\title{
MPB Regimen
}

National Cancer Institute

\section{Source}

National Cancer Institute. MPB Regimen. NCI Thesaurus. Code C64836.

A regimen consisting of melphalan, prednisone and bortezomib used for the treatment of plasma cell myeloma. 